\title{
FRETTING WEAR ANALYSIS OF DIFFERENT TUBE MATERIALS USED IN HEAT EXCHANGER TUBE BUNDLE
}

\author{
Tasawar Abbas ${ }^{1 *}$, Shahab Khushnood ', Luqman Ahmad Nizam', Muhammad Usman ${ }^{1}$ \\ 1 University of Engineering and Technology Taxila, Mechanical and Aeronautical Engineering Department, \\ 47080 Taxila, Pakistan \\ E-mails: tasawarabbas231@gmail.com, shahab.khushnood@uettaxila.edu.pk, engr.luqmannizam@gmail. \\ com, muhammadusman3051@gmail.com
}

Received: 2017.08.19

Accepted: 2017.11.01 Published: 2017.12.05

\begin{abstract}
Research on heat exchange has been carried out for more than five decades because of its importance in process industries and power generation plants. Heat exchanger experiencing cross flow are vulnerable to flow-induced vibration. This vibration causes the interaction of tubes with the baffle resulting in a fretting wear of the tubes. The present study focuses on fretting wear analysis of different tube materials. Fretting wear tests were performed on aluminum, copper and stainless steel instrumented central tubes against mild steel baffle. For each tube material the tests were performed for three different test durations i.e. 60 minutes, 120 minutes and 180 minutes at a cross flow velocity of $0.55 \mathrm{~m} / \mathrm{s}$. It was observed that vibrational amplitude of the flexible test tube is affected by its weight. A scanning electron microscope was used to analyze and measure the sizes of wear scar. The results indicated that wear loss in case of aluminum tube is the highest while that in case of stainless steel tube is the lowest.
\end{abstract}

Keywords: fretting wear; flow induced vibration; flexible tube; cross flow velocity

\section{INTRODUCTION}

Being the fundamental part of the process industries and power plants, heat exchanger plays a critical role in effective and safe operation of process and power generation industries. Baffles/ support plates are provided with the heat exchanger to enhance the heat transfer rate and in some cases to guide fluid flow. The increase in shell side fluid velocity causes vibrations in the tubes which may lead to tube-to-tube strike and fretting wear, if of high amplitude. One of the major problems associated with the steam generators used in nuclear power plants is the fluid-structure interaction (FSI). Steam generator's tubes experiencing cross flow are extremely vulnerable to FIV. Fretting may occur because of these vibrations on the surface of the tubes in contact with the supporting plate, which may lead to the failure of tube bundle of heat exchanger. Heat exchanger needs to be shut down because of tube bundle failure and the entire process demanding costly damage repair. Fretting is a complicated phenomenon that is associated with the oscillatory motion of small amplitude between the surfaces in contact with each other [25]. Fretting may cause the calamitous failure of the part in service, therefore, assessment of the wear caused by fretting is necessary in order to predict the useful service life of the parts being used in many engineering applications [19]. Cracking and wear are considered to be the most fatal damage under fretting [3, 22].

Many nuclear power plants have reported degradation in steam generator/heat exchanger tube materials because of fretting, which needs to be considered of practical significance for safe, economical and consistent operation of nuclear power industries/plants $[2,24]$. These degradations in heat exchangers take place because of the combined effect of the vibration induced by the 
flow and the small clearance in-between tube and its support material or plate. A lot of research work has been done to assess the fretting wear characteristics of materials used in nuclear engineering under various mechanical parameters (frequency, displacement amplitude, normal force, etc.), service condition and materials structures $[7,14,16$, 26]. Gross slip and partial slip regime are the two major domains that can be recognized according to the contact conditions. Considering the material reaction against fretting loading, it is observed that wear occurs in the gross slip, while cracking is usually identified in partial slip [17, 23]. Material strength is significantly degraded by the crack due to fatigue stress, consequently reducing the service life of the component. Recently, considering the residual life of the tube, some research work is carried out to calculate fretting wear coefficient $[1,12]$. Experimental studies related to the fretting wear SG tubes have been carried out since 1970 [8]. J. Li analyzed fretting wear behavior of Inconel 600(nickel-chromium alloy) with various number of fretting cycles in dry conditions and at room temperature. He concluded that Inconel 600 shows a change in behavior with the increase in number of cycles. From the results he obtained that wear scar depth, wear volume and plastic regime at the edge of the scar first increased slowly with the increase in number of cycles and then showing a significant increase, consequently resulting in transformation of wear mechanism from adhesive wear to mixture of delamination wear and oxidation. With the further increase in number of cycle, stick zone disappeared resulting in the formation of tribologically transformed structure (TTS) which was later on destroyed by the micro rack formation and propagation [15]. In order to reduce the damage caused by wear in tube's material, wear mechanisms should be analyzed at different test parameters such as applied normal load, temperature, counterpart material and sliding amplitude. Most of the research work related to tube fretting wear focuses on the calculation of wear coefficients for different test condition $[4,6,20]$. To have the complete understanding of wear characteristic, steam generator wear model should be considered not only for the evaluation of wear coefficient, but also for contact surfaces wear mechanism. Sliding wear test of SG tube materials performed against stainless steel concluded that, by increasing sliding amplitude and normal load the, there was a rapid increase in wear rate of mill annealed Inconel
600( nickel-chromium based alloy), but wear transition happened only in thermally treated Inconel 690(nickel-chromium alloy) above the $15 \mathrm{~N}$ applied normal load and amplitude of 30 micrometer. SEM analysis of worn surfaces showed that particle removal of the tube materials consists of different mechanisms. The wear rate for thermally treated Inconel 600 is lower as compared to mill annealed Inconel 600 , but wear transition occurs only in thermally treated Inconel 600 and not in the mill annealed Inconel 600 [13].

P.L. Ko, N.J. Fisher and M.J. Pettigrew, et al. conducted researches to analyze the influence of fretting wear on the tubes of steam generator. They concluded that wear as result of impact was smaller, as compared to wear that occurs as a result of pure sliding for the testing of anti-vibration bar (AVB) with $403 \mathrm{SS}$ and Inconel 600 [9, 11, 20, 21]. Years later, wear regimes were classified into four types, stick, gross slip, mixed slip and stick and sliding by Vingsbo and Soderberg [23]. There is no change in wear coefficient with increase in sliding amplitude in sliding regime. Fretting wear is affected by some elements including frequency, normal load, amplitude and environment (dry, wet, temperature etc.). Amplitude and normal load are considered as the two most important elements that has a direct effect on the form and extent of damage caused by the fretting wear. Generally, there is a difference in effects of normal load in fretting wear test and sliding wear test but all the four regimes of the fretting map are affected directly by the amplitude. Usually, steam generator wear is affected by the different parameters directed by the flow of the fluid, e.g., vibration frequency, type of tube motion and the impact force acting at the supports. It is also governed through mechanical design, e.g. contact area between tube and support, material combination, operating temperature and clearance between tube and support [8]. Roger [18] and Ko [10] carried out experimental and analytical studies on the interaction of tube and support, relating damage of these sites caused by fretting wear due to FIV initiated by different mechanism like fluid elastic instability, periodic vortex shedding and random excitation as a result of fluid turbulence. Mechanisms of vibration induced by flow and its effects on the components of heat exchanger were reviewed by Goyder [5]. High cross flow velocity inside the shell was observed to the main reason of the tubes vibration with their supports. It was observed that along with the high velocity the in- 
tube fluid was the other factor causing the tube instability by adding more weight. Multiphase buffeting, vortex shedding and fluid elastic instability were found to be responsible for imparting vibrational effects and for the relative motion between tube and its support.

The present study focuses on fretting wear analysis of different tube materials. The main purpose of this research work is to investigate the wear effects on a flexible tube in a tube bundle caused by FIV. These wear effects were analyzed for different test duration at the constant cross flow velocity.

\section{EXPERIMENTAL SETUP AND METHODOLOGY}

The experiment was carried out on the low speed closed channel water tunnel with a velocity in the test section ranging from 0.18 to 0.55 $\mathrm{m} / \mathrm{s}$ which was designed and developed by FIV research group of mechanical engineering department at university of engineering and technology Taxila. The converging diverging part of the test rig is metallic and test section is made of acrylic plastic material. The experimental setup is presented in Figure 1.

The test section has dimensions of $200 \mathrm{~mm} \mathrm{x}$ $100 \mathrm{~mm}$ and is made up of acrylic plates that are screwed together. The closed loop water flow is maintained by a $10 \mathrm{HP}$ centrifugal pump and a 200 gallons water storage tank. The main valve and bypass valve are provided to control water velocity in the test section up to its maximum value with each increment of $0.09 \mathrm{~m} / \mathrm{s}$. An ultrasonic flow meter (Doppler type) was installed in the test section to measure the upstream velocity of the tube bundle. Due to its working nature, the flow measuring transducer was mounted on the outer surface of the test section. Water is drawn from the water storage tank, accelerated and passed through the test section. The carefully designed flow straightener ensure a uniform velocity distribution with little turbulence in the test section. The tube bundle under investigation is mounted in the test section while the flow medium is set in motion, and thus the desired flow around the tubes in a tube bundle is generated.

Tubes made of aluminum have been used to form parallel triangular tube bundle. Figure 2 shows the tube bundle configuration used in this experiment $\mathrm{P} / \mathrm{D}$ 1.45. The black dot represents the position of the instrumented tube, $\mathrm{Y}$ shows the stream-wise whereas $X$ shows the transverse direction. The monitored tube was instrumented with tri-axial accelerometer to monitor vibrations in stream-wise ( $\mathrm{Y}$, in the direction of flow) and in transverse (X, perpendicular to flow) directions with natural frequency of a tube, tuned to nearly $8 \pm 0.1 \mathrm{~Hz}$. The monitored tube was tightened by means of $0.2 \mathrm{~mm}$ thick piano wire and a tensioning mechanism to adjust the natural frequency of

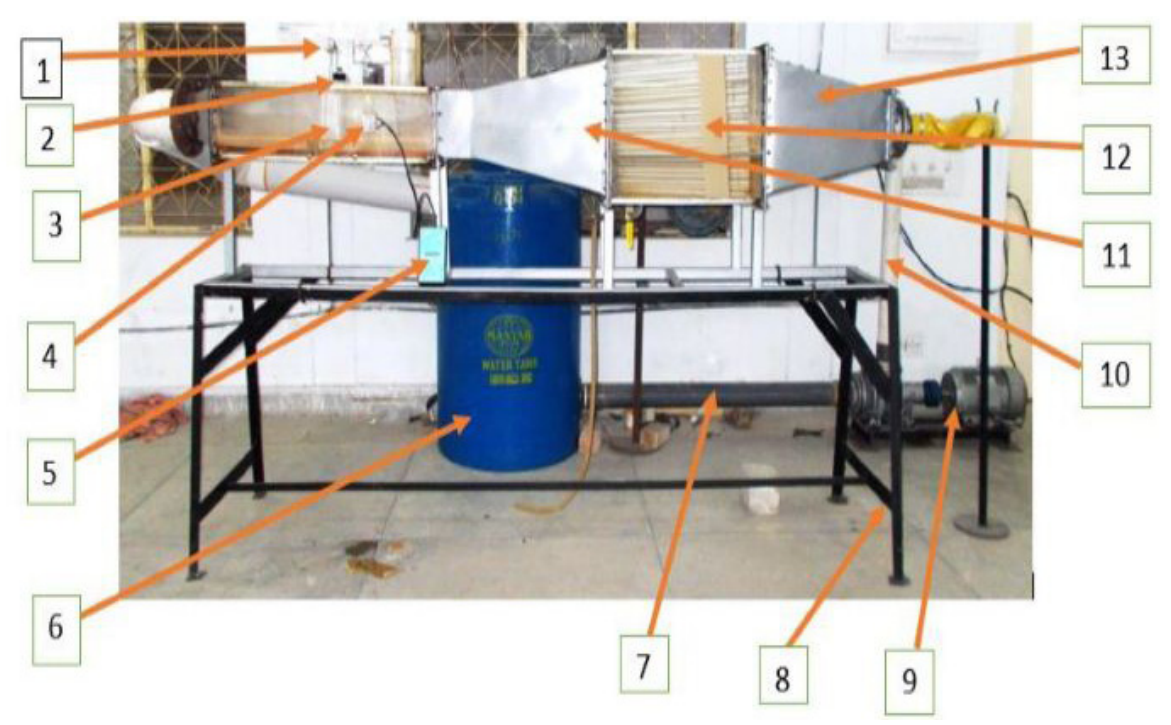

Figure 1. Low speed closed channel Water tunnel

1- Tube Bundle; 2- G-Link Tri-Axial Accelerometer; 3- Test Section; 4- Flow Meter Transducer; 5- Doppler Flow Meter; 6- Water Tank; 7- Pump Suction Line; 8- Water Tunnel Stand; 9- 10 HP Motor with Centrifugal Pump; 10- Pump Delivery Line; 11-Convergent Section; 12- Flow Straightener Section; 13- Divergent Section 
monitored tube. The accelerometer was mounted on the top of the monitored tube in order to capture the vibration response of the tube in cross flow. The whole assembly was supported by two acrylic plates, one used to hold the rigid tubes and the other used to hold the tensioning mechanism Figure 3. Table A presents specifications of the test tubes and tube bundle.

The plates holding the tube bundle were designed and manufactured in such a way as to fit in the space provided in the test section of the water tunnel. Typically, the plates had dimension of 200 $\mathrm{mm} \times 90 \mathrm{~mm}$. Before performing the tests, the plates were held fixed in the test section in order to avoid any error. After the plates were fixed, the tests were performed on three different tubes (Aluminum, Copper, Stainless steel) each tube having the same dimension. The cross flow velocity for each tube was kept constant i.e. $0.55 \mathrm{~m} / \mathrm{s}$.
For each tube material tests were performed for three time spans i.e. 1 hour, 2 hours and 3 hours. After the tests were performed the wear affected parts of the tubes were cut into small pieces and total of 9 samples were prepared for three different time spans (60 minutes, 120 minutes, 180 minutes). A scanning electron microscope (SEM) was used to analyze and measure the sizes i.e. length and width of wear scar created on the tubular surface during experimentation Figure 4 . In order to measure the depth, a new technique has been used. Various samples of known wear depth on similar tube material was test under SEM and cross surface profiles has been captured. Based on these measurements a calibration curve was plotted. From this curve, a conversion factor was deduced i.e. $(1 \mathrm{Adu}=0.3055 \mu \mathrm{m})$. Based on this conversion factor, the wear depth of wear scars formed in the experiments was estimated.

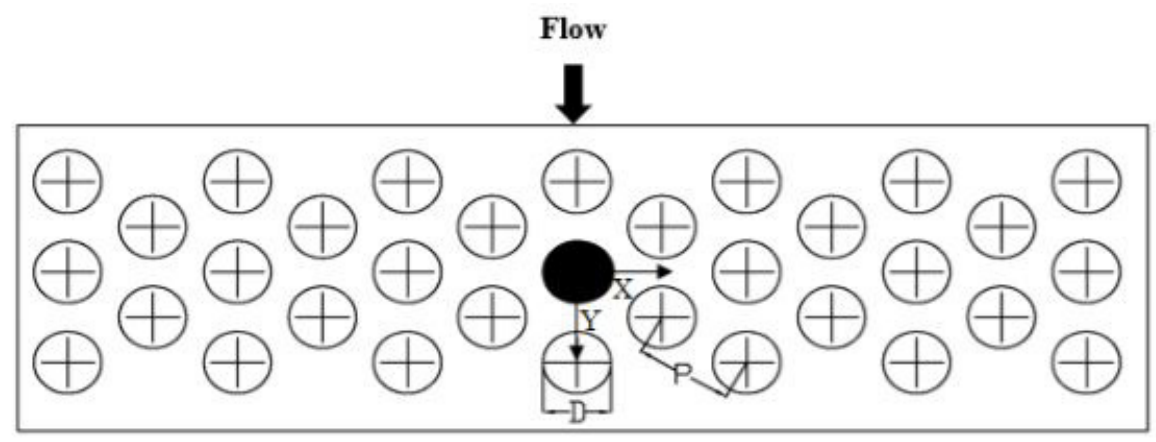

Figure 2. Tube Bundle Configuration with $\mathrm{P} / \mathrm{D}$ of $1.45(\mathrm{X}=$ Transverse, $\mathrm{Y}=$ Stream-wise $)$

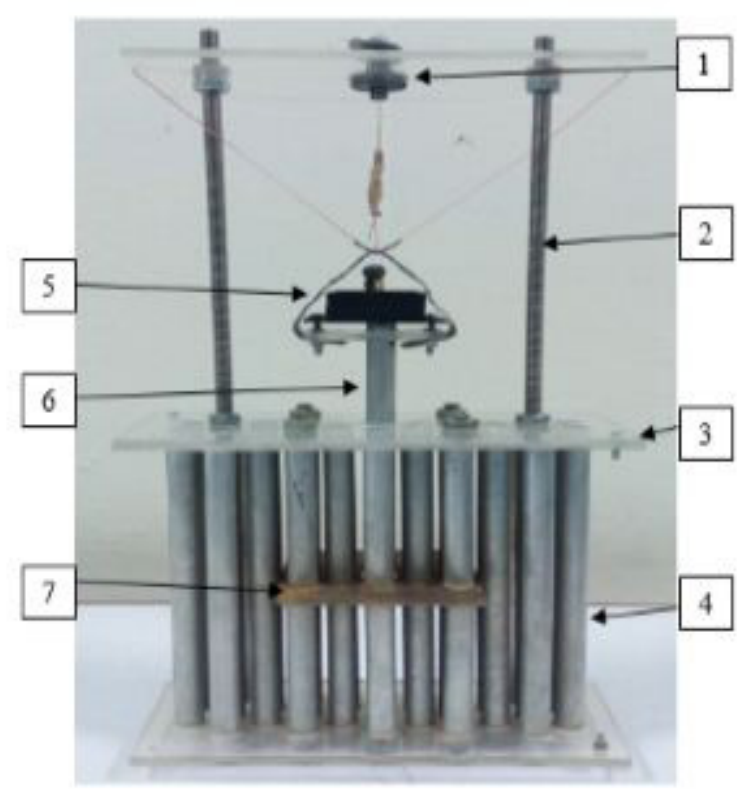

Figure 3. Photograph of Tube Bundle (1) Tensioning mechanism (2) Support stud (3) Support plates (4) Rigid tubes (5) Accelerometer (6) Monitored tube (7) Baffle 
Table A: Specifications of tube bundle and test tubes

\begin{tabular}{|l|l|l|l|}
\hline Tube material & Aluminum & Copper & Stainless Steel \\
\hline Type of tube array & Parallel triangular & Parallel triangular & Parallel triangular \\
\hline Mass of the tube & $56.13 \mathrm{~g}$ & $186.28 \mathrm{~g}$ & $160 \mathrm{~g}$ \\
\hline P/D ratio & 1.45 & 1.45 & 1.45 \\
\hline Tube outer/inner diameter & $12.7 / 11.5 \mathrm{~mm}$ & $12.7 / 11.5 \mathrm{~mm}$ & $12.7 / 11.5 \mathrm{~mm}$ \\
\hline Tube length & $228 \mathrm{~mm}$ & $228 \mathrm{~mm}$ & $228 \mathrm{~mm}$ \\
\hline Modulus of Elasticity & $69000 \mathrm{MPa}$ & $117000 \mathrm{MPa}$ & $180000 \mathrm{MPa}$ \\
\hline Tube density & $2.7 \mathrm{~g} / \mathrm{cm}^{3}$ & $8.96 \mathrm{~g} / \mathrm{cm}^{3}$ & $7.7 \mathrm{~g} / \mathrm{cm}^{3}$ \\
\hline Width of Baffle & $0.6 \mathrm{~mm}$ & $0.6 \mathrm{~mm}$ & $0.6 \mathrm{~mm}$ \\
\hline Baffle whole diameter & $15 \mathrm{~mm}$ & $15 \mathrm{~mm}$ & $15 \mathrm{~mm}$ \\
\hline Tube to baffle whole clearance & $1.15 \mathrm{~mm}$ & $2.3 \mathrm{~mm}$ & $2.3 \mathrm{~mm}$ \\
\hline
\end{tabular}

\section{RESULTS AND DISCUSSION}

\section{Vibration analysis}

Vibration signal of the three test tubes i.e. aluminum tube, copper tube and stainless tube for stream-wise and transverse direction are presented in Figure 5 while the RMS response of the three test tubes at the same velocity is presented in Figure 6.

It is evident from Figure 5 (a) that amplitude of vibration for the aluminum tube is higher, as compared to copper and stainless steel tubes apart from the fact that the amplitude of vibration in transverse direction is a little higher than streamwise amplitude for all the three test tubes. This difference between transverse and stream-wise amplitude is significant in case of aluminum tube. Figure 6 presents a more elaborated view of tubes response. As it can be seen that the transverse as well as stream-wise amplitude of aluminum tube is highest while the copper tube RMS amplitude is the lowest at the same velocity and for the same test time. This difference in amplitude is because of difference in masses of the tubes, the higher the mass of vibrating tube lesser will be its amplitude of vibration. Since mass of the aluminum tube is least among all the three tubes that is why its amplitude of vibration is highest, while mass of copper tube is highest that is why its amplitude of vibration is lowest both in stream-wise as well as in transverse direction.

Although in first glance, it looks that vibration response of the tube is represented by the

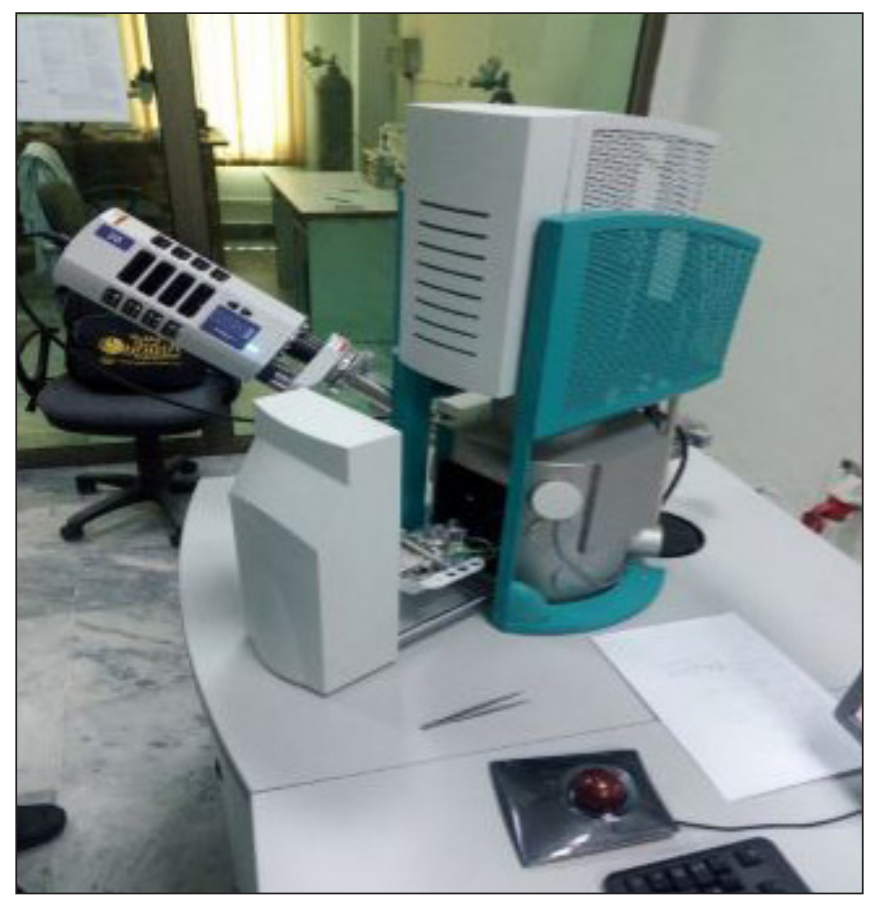

Figure 4. Photograph of Scanning Electron Microscope (SEM) 
a)
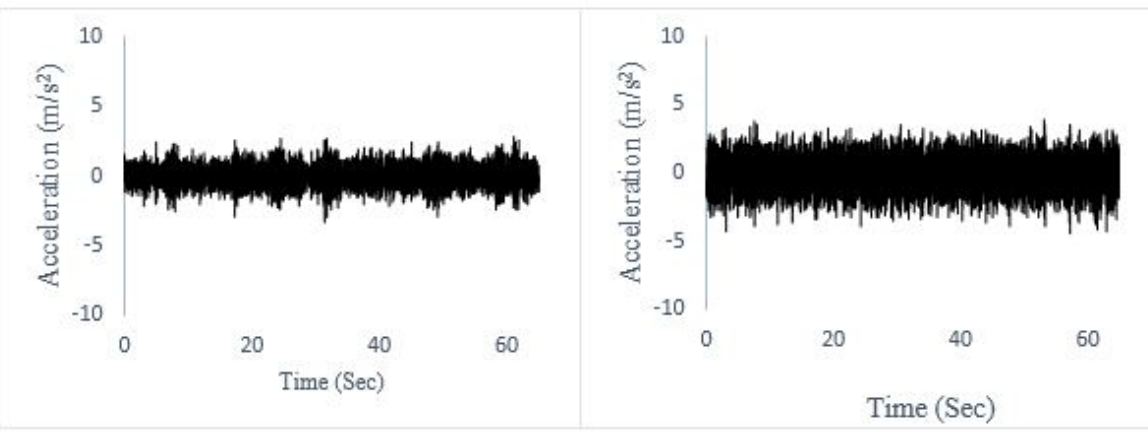

Stream-wise

Transverse

b)

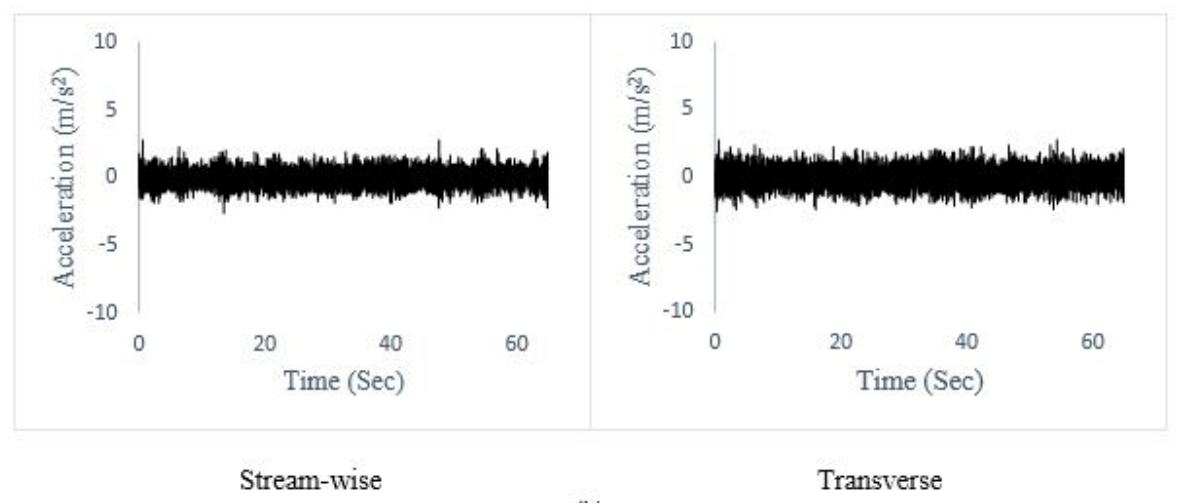

c)

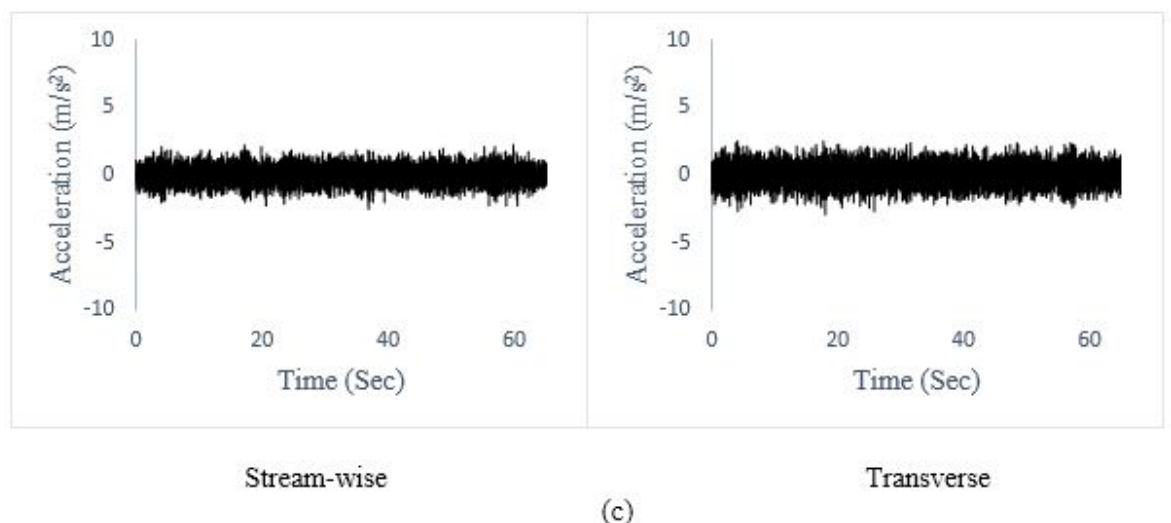

(c)

Figure 5. Vibration signals of the tubes (a) Aluminum tube (b) Copper tube (c) Stainless steel tube

transverse amplitude value but in fact it is due to impacting of tube with the inner surface of the baffle hole. This causes the significant wear of the tube at higher velocities that is predominantly in transverse direction. So there is a strong relation among wear produced in the tube and acceleration amplitude, which is discussed in the later section.

\section{Sem images and wear profile}

The scanning electron microscope (SEM) images and the wear profiles of three different test tubes (aluminum, copper, stainless steel) at the same cross flow velocity and for the same test time are presented in the Figure 7. The investigation of wear profiles and corresponding characteristics conclude that wear width tends to increase with the duration of interaction of tube with the baffle. It was also observed that wear depth follows the same trend.

As it is evident from the wear profiles, which are highlighted by red circles, there are relatively small profile peaks visible for copper and stainless steel tubes Figure $7(b, c)$. This corresponds to a comparatively small wear depth for copper and stainless steel tubes. But in case of aluminum tube, a sharp profile relatively of higher magnitude peaks can be seen that shows the higher wear 


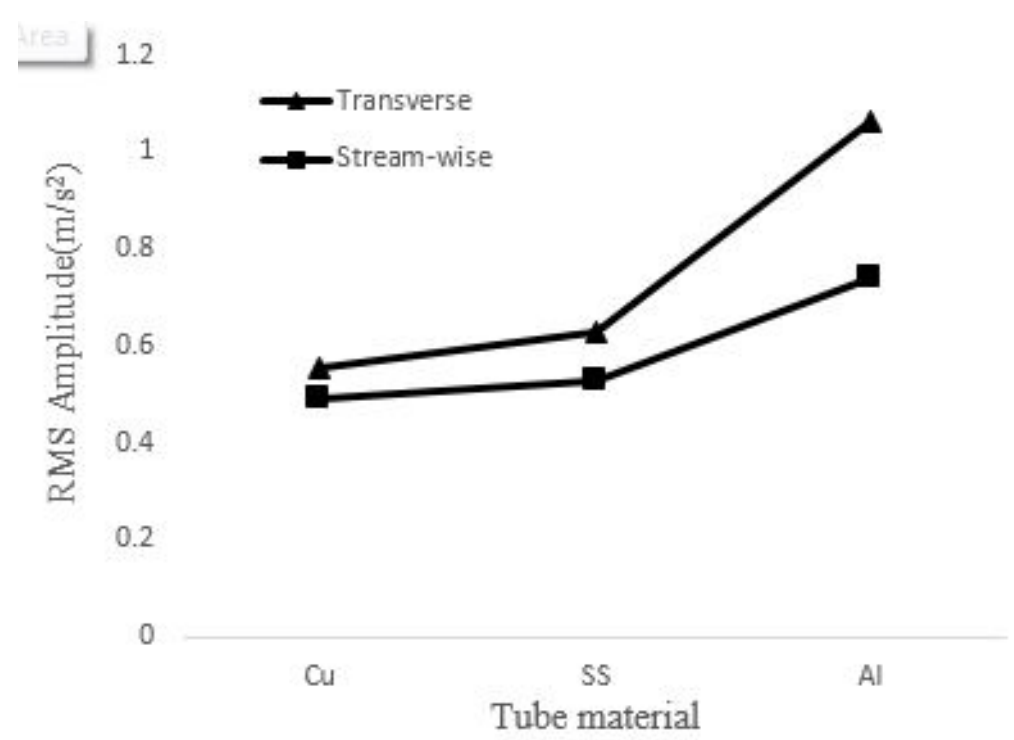

Figure 6. RMS amplitude response of tubes

depth for aluminum tube Figure 7 (a) concluding that the longer the wear profiles higher will be the depth of wear scar and vice versa.

\section{INVESTIGATION OF WEAR SIZES}

\section{Wear length}

The variation of length with the time for three different test tubes are presented in the Figure 8. It can be seen that wear length increases gradually with the increase in test time. It can be seen that wear length in case of aluminum tube increases at a moderate rate from $1609.5 \mu \mathrm{m}$ for the duration of 60 minutes to $2176.04 \mu \mathrm{m}$ for the duration of 120 minutes. However, wear length shows a significant rise for the duration of 180 minutes with the maximum wear length reaching to $2996.9 \mu \mathrm{m}$.

The same trend can be seen for the wear length of copper as well as stainless steel tube. A possible description for such behavior of length increase with the duration is the third body abrasion that is associated with the shape of contact. Since in order to have a contact between tube ant baffle, the clearance between tube and support should be narrow. The narrow clearance results in the harder dispersion of the wear particles generated from contact surface. These particles may gather for some time before complete dispersion. During the accumulation these wear particles act as a third body, what results in extra abrasive wear on the clearance region. The width of the wear scar almost follows the same trend. It is also very important to note that slope of the trend line goes on increasing with the increase in time of interaction of tube with the baffle. This difference in the slope of the trend line with the time shows that wear length increases significantly as the duration of contact between tubes and baffle increases.

\section{Wear area}

Figure 9 presents the variation of wear area with the increase in the test duration for all three test tubes. Wear area is calculated by simply multiplying the wear length with wear scar width for each tube at specified duration. As it can be seen that up to duration of 120 minutes wear area in case of all the three tubes increases at a moderate rate with the test time, but after 120 minutes wear area increases significantly up to 180 minutes. Since the area is obtained simply by multiplying the wear scar length and width of wear scar for each specimen at specified test time, so it follows the same trend as that of scar length and width. Since cross flow velocity of water flowing outside the tube bundle is constant for all the three test tubes, so it can be seen that at the same test duration there is a major difference in worn scar area for three test tubes. The worn scar area of aluminum tube is much larger than copper and stainless steel tube. On the hand worn scar area of stainless steel tube is almost negligible, as compared to copper and aluminum tube. This difference of area at the same test duration is attributed to the mechanical properties of the tube materials. 
a)
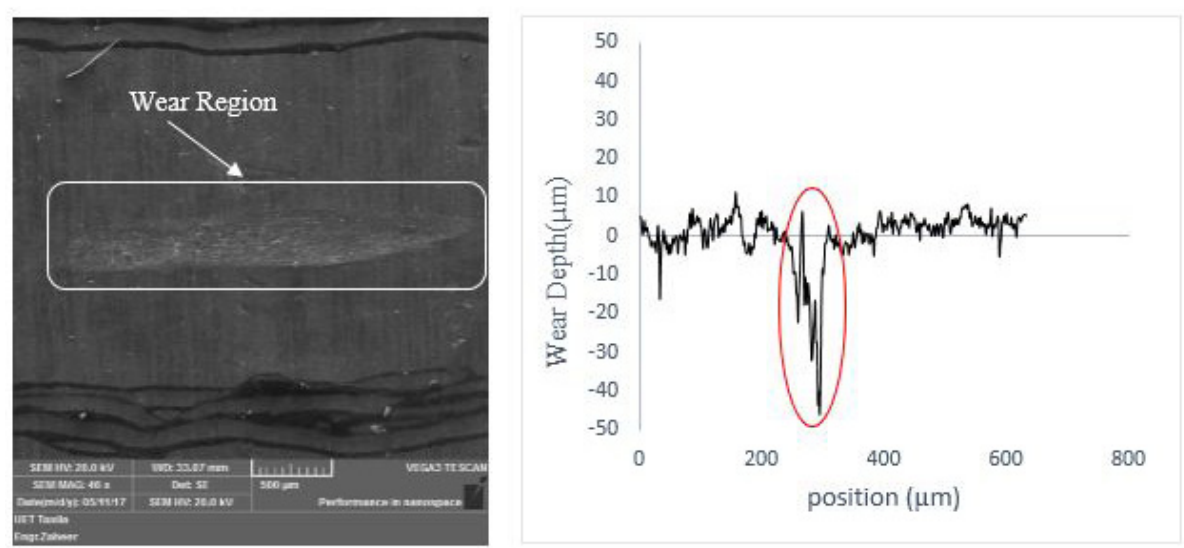

b)
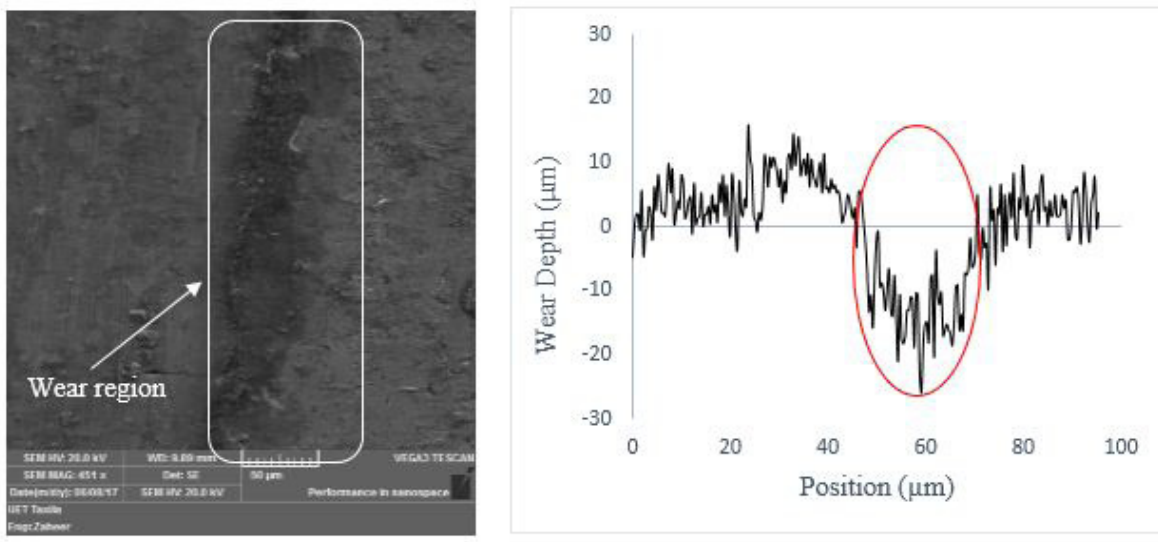

c)
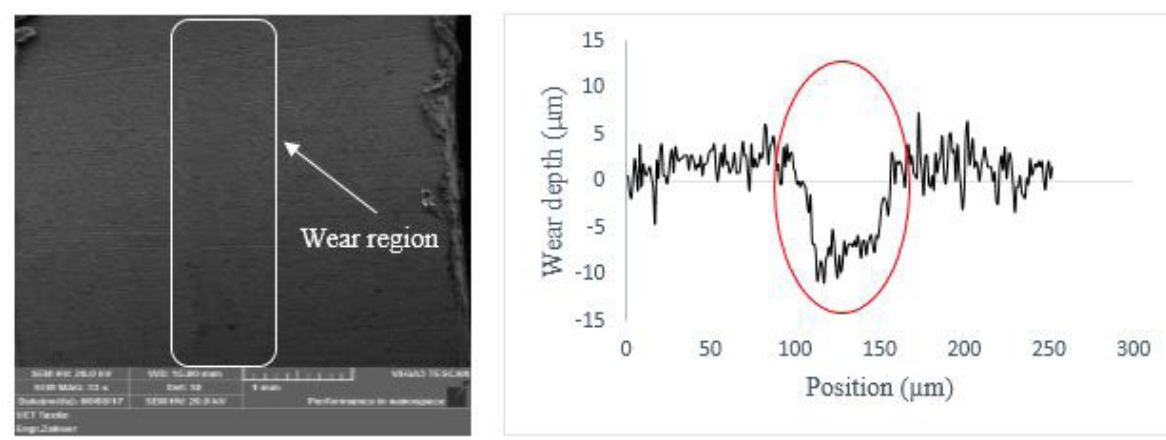

Figure 7. Scanning Electron Microscope (SEM) images and wear profiles of the tubes (a) Aluminum tube (b) Copper tube (c) Stainless steel tube

\section{WEAR VOLUME}

Based on the depth of wear scars, which is estimated by the analysis of wear profiles from the scanning electron microscope (SEM) images, a wear volume analysis was carried out to find out the volume loss for each specimen at different test duration. The basic purpose of this analysis was to investigate a relationship between wear volume and test duration at the constant cross flow velocity for three different test tubes. Figure 10 presents the trend of wear volume with the test duration for three different test tubes.
The analysis suggests that wear volume exhibits increasing trends with the increase in test duration. It is evident from Figure 10 that the rate at which wear volume increases from 60 to 120 minutes is less than the rate at which wear volume increase from 120 to 180 minutes, with the wear volume of $4.818 \times 10^{-3} \mathrm{~mm}^{3}$ and $10.347 \times 10^{-3}$ $\mathrm{mm}^{3}$ at 60 and 120 minutes respectively in case of $\mathrm{Al}$ tube but after the test duration of 120 minutes wear volume increases more rapidly with the test time up to 180 minutes where the maximum wear volume reaches $20.089 \times 10^{-3} \mathrm{~mm}^{3}$ is almost double the value of wear volume at 120 
a)

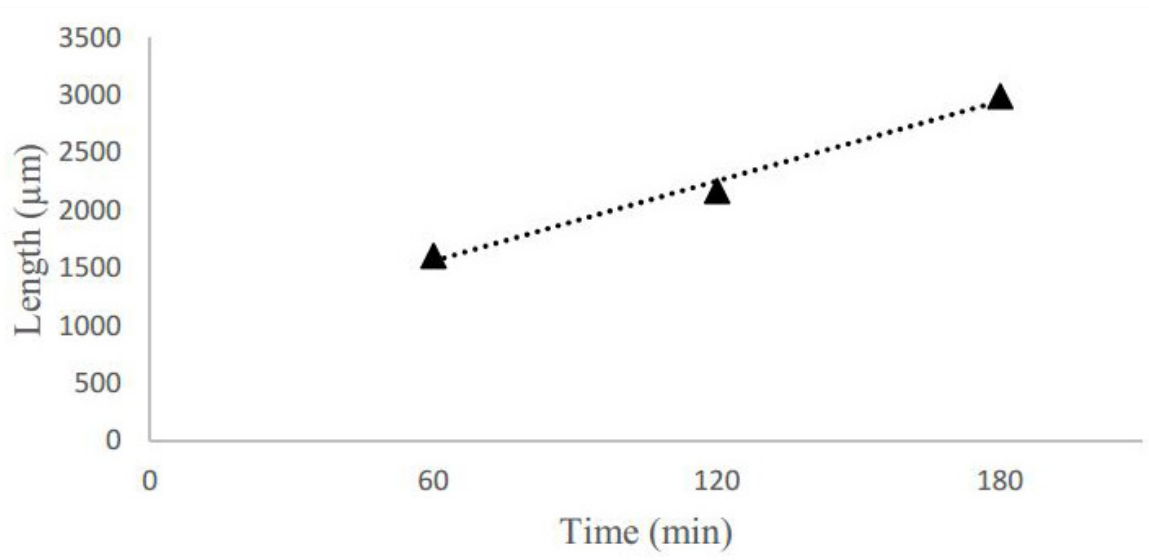

b)

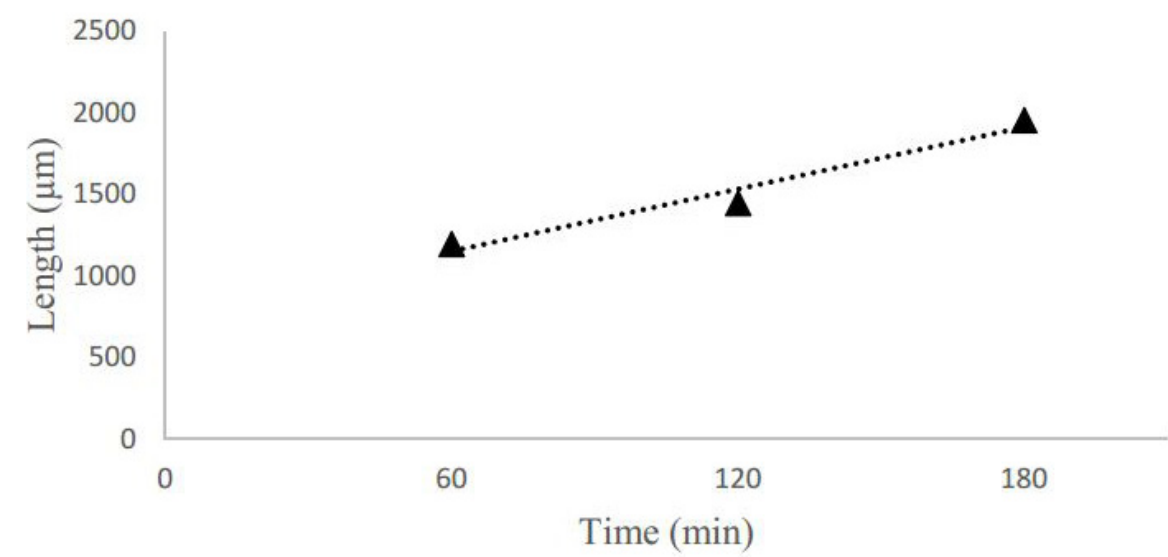

c)

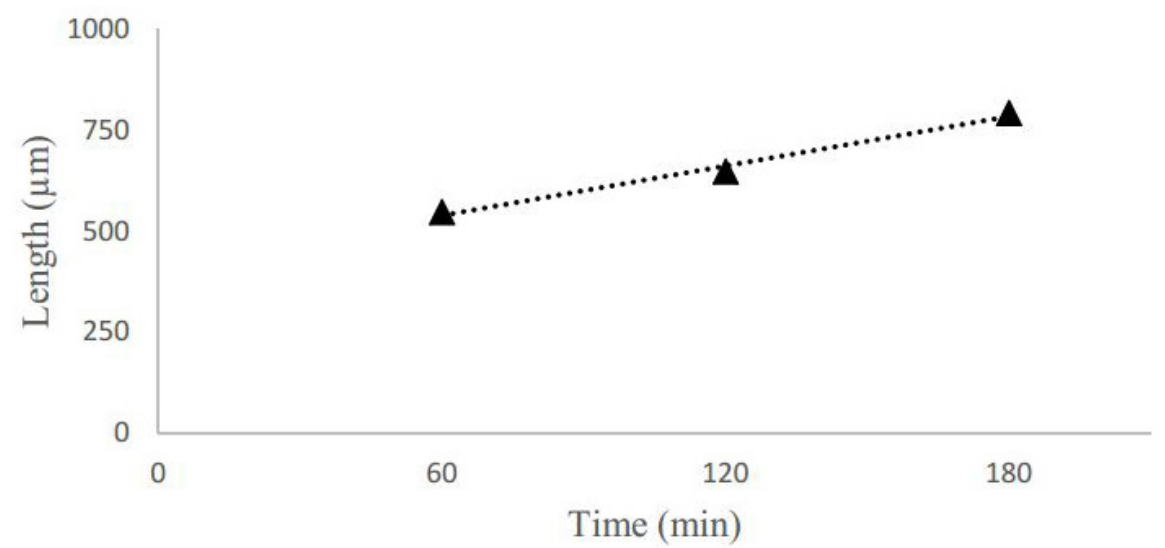

Figure 8. Variation of Wear length vs. test duration (a) Aluminum tube (b) Copper tube (c) Stainless steel tube

minutes. Copper and stainless steel tubes follow the same trend. It is evident from Figure 10 that wear volume of the stainless steel tube is almost negligible, as compared to aluminum and copper tubes, provided the same cross flow velocity and for the same test duration, while the wear volume of aluminum tube is much higher than the copper and stainless steel tubes. This difference of volume loss for three different test tubes experienc- ing the same cross flow velocity and for the same test duration is attributed to hardness of the tube materials. Among all the mechanical properties of the materials hardness is one of critical factor that affect the fretting wear most. Hardness of a material is directly related to its wear resistance, more the harder material is more it will be wear resistant and vice versa. Since among all the three test tubes stainless steel is much harder than copper 


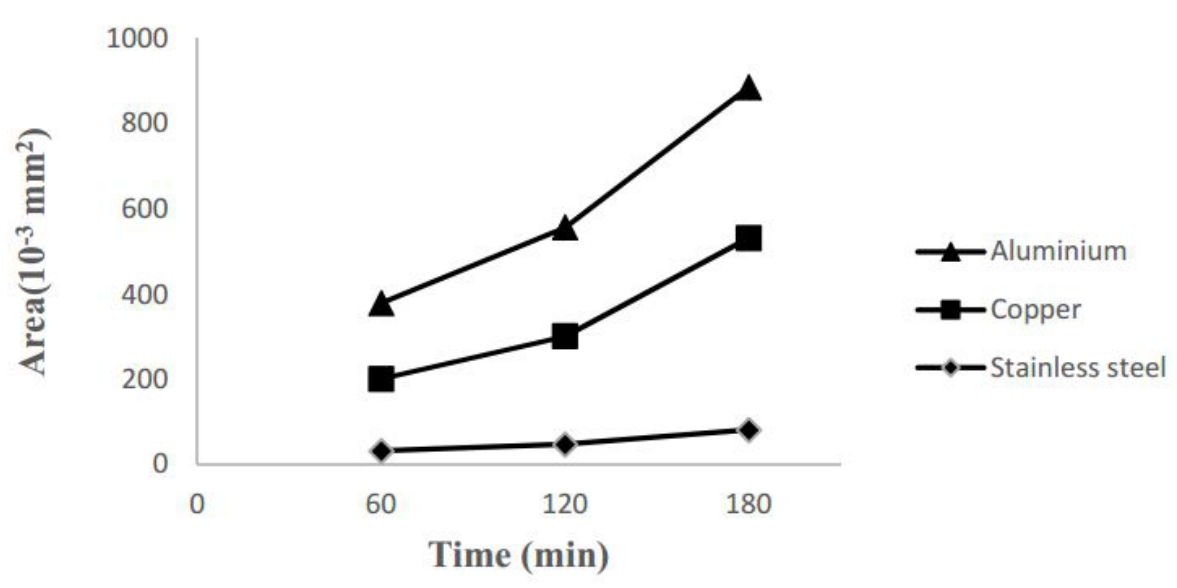

Figure 9. Wear area of tubes with increase in test duration

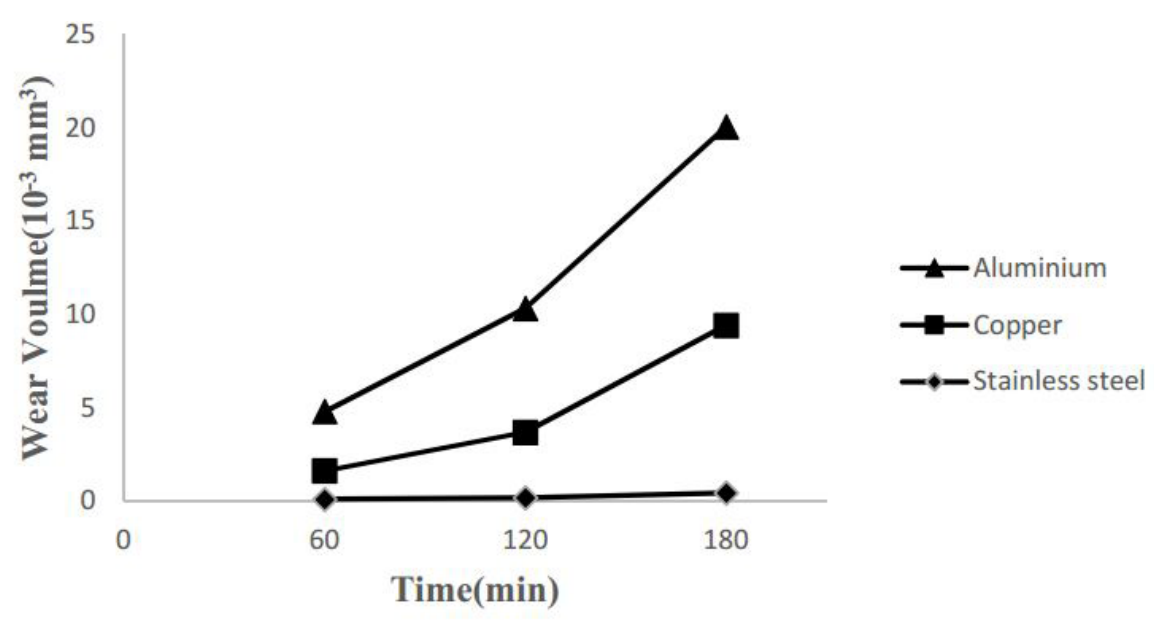

Figure 10. Wear volume of tubes with increase in test duration

and aluminum that is why it is less susceptible to wear while aluminum is a softer material among all the three test tubes so it is more vulnerable to wear as compared to other two.

Based on the current analysis a linear trend cannot be expected between wear volume and duration of tube interaction with the baffle. A comprehensive study is required, which should include the wear analysis for long duration in order to confirm the behavior of wear volume.

\section{CONCLUSION}

The fretting wear tests were performed on an instrumented central tube in a parallel triangular tube bundle for three different tube materials (aluminum, copper, stainless steel). The experiments were conducted for various test durations at a cross flow velocity of $0.55 \mathrm{~m} / \mathrm{s}$ in a closed loop water tunnel. The conclusions can be summarized as follows:
1. From the vibration analysis, it was observed that the amplitude of vibration in transverse direction was more than that in stream-wise direction, which suggests that significant wear occurs predominantly in transverse direction (perpendicular to flow).

2. The results indicated that with the increase in test duration wear scar length, wear area and wear volume increase moderately at first and then shows a significant increase with the further increase in test duration due to third body abrasion associated with the shape of contact between tube and the baffle.

3 . The wear volume analysis indicated that volume loss of aluminum tube was the highest, whereas that in case of aluminum tube was the lowest but volume loss in case of copper tube was the in-between these two. This analysis suggests that being a softer material, aluminum is less resistant to wear as compared to copper and stainless steel. 


\section{Acknowledgment}

Authors are grateful to the "Faculty and helping staff of the composite lab in Mechanical engineering department of University of Engineering and Technology Taxila, Pakistan.

\section{REFERENCES}

1. Attia, M. and E. Magel, Experimental investigation of long-term fretting wear of multi-span steam generator tubes with U-bend sections. Wear, 1999. 225: p. 563-574.

2. Attia, M.H., Fretting fatigue and wear damage of structural components in nuclear power stationsFitness for service and life management perspective. Tribology International, 2006. 39(10): p. 1294-1304.

3. Blanchard, P., et al., Material effects in fretting wear: application to iron, titanium, and aluminum alloys. Metallurgical Transactions A, 1991. 22(7): p. $1535-1544$.

4. Fisher, N., A. Chow, and M. Weckwerth, Experimental fretting-wear studies of steam generator materials. Transactions-American Society Of Mechanical Engineers Journal Of Pressure Vessel Technology, 1995. 117: P. 312-320.

5. Goyder, H., An assessment method for unstable vibration in multispan tube bundles. Journal of fluids and structures, 2003. 18(5): p. 555-572.

6. Guerout, F.M. and N.J. Fisher, Steam generator fretting-wear damage: a summary of recent findings. Transactions-American Society Of Mechanical Engineers Journal Of Pressure Vessel Technology, 1999. 121: P. 304-310.

7. Kim, D.-G. and Y.-Z. Lee, Experimental investigation on sliding and fretting wear of steam generator tube materials. Wear, 2001. 250(1): p. 673-680.

8. Ko, P., Experimental studies of tube fretting in steam generators and heat exchangers. ASME J. Pressure Vessel Technol, 1979. 101: p. 125-133.

9. Ko, P., Heat exchanger tube fretting wear: review and application to design. J. Tribology(Trans. ASME), 1985. 107(2): p. 149-156.

10. Ko, P., Metallic wear-a review with special references to vibration-induced wear in power plant components. Tribology International, 1987. 20(2): p. 66-78.

11. Ko, P. and H. Basista, Correlation of support im- pact force and fretting-wear for a heat exchanger tube. Journal of pressure vessel technology, 1984. 106(1): p. 69-77.

12. Lee, Y.-H., et al., A comparative study on the fretting wear of steam generator tubes in korean power plants. Wear, 2003. 255(7): p. 1198-1208.

13. Lee, Y.-H., et al., A study on wear coefficients and mechanisms of steam generator tube materials. Wear, 2001. 250(1): p. 718-725.

14. Li, J., et al., Effect of grain size and hardness on fretting wear behavior of Inconel 600 alloys. Tribology International, 2015. 81: p. 215-222.

15. Li, J., et al., Evolution of wear damage in Inconel 600 alloy due to fretting against type 304 stainless steel. Wear, 2016. 346: p. 15-21.

16. Lim, M.-K., S.-D. Oh, and Y.-Z. Lee, Friction and wear of Inconel 690 and Inconel 600 for steam generator tube in room temperature water. Nuclear Engineering and Design, 2003. 226(2): p. 97-105.

17. Ödfalk, M. and O. Vingsbo, An elastic-plastic model for fretting contact. Wear, 1992. 157(2): p. 435-444.

18. Rogers, R. and R. Pick, On the dynamic spatial response of a heat exchanger tube with intermittent baffle contacts. Nuclear Engineering and Design, 1976. 36(1): p. 81-90.

19. Shen, M., et al., Dual-rotary fretting wear of 7075 alloy in media of oil and water. Wear, 2013. 301(1): p. 540-550.

20. Taponat, M. and M. Zbinden, Wear studies of materials for tubes and antivibration bars in nuclear steam generators. Journal of pressure vessel technology, 1996. 118: p. 287.

21. Taylor, C., et al., Vibration damping in multispan heat exchanger tubes. Journal of pressure vessel technology, 1998. 120(3): p. 283-289.

22. Vincent, L., et al., Mechanics and materials in fretting. Wear, 1992. 153(1): p. 135-148.

23. Vingsbo, O. and S. Söderberg, On fretting maps. Wear, 1988. 126(2): p. 131-147.

24. Wade, K.C., Steam generator degradation and its impact on continued operation of pressurized water reactors in the United States. Energy Information Administration/Electric Power Monthly, 1995. 66.

25. Waterhouse, R. and D. Taylor, Fretting debris and the delamination theory of wear. Wear, 1974. 29(3): p. 337-344.

26. Zhang, H., et al., Effect of precipitated carbides on the fretting wear behavior of Inconel 600 alloy. Wear, 2014. 315(1): p. 58-67. 